\title{
Amino Acid Side Chains Buried Along Intersubunit Interfaces in a Viral Capsid Preserve Low Mechanical Stiffness Associated to Virus Infectivity
}

Pablo José P. Carrillo, María Medrano, Alejandro Valbuena, Alicia Rodríguez-Huete, Milagros Castellanos, Rebeca Pérez, and Mauricio G. Mateu*

Centro de Biología Molecular "Severo Ochoa" (CSIC-UAM), Universidad Autónoma de Madrid, c/ Nicolás Cabrera 1, Cantoblanco, 28049 Madrid, Spain

*Address correspondence to: mgarcia@cbm.csic.es 


\begin{abstract}
Single-molecule experimental techniques and theoretical approaches are revealing that important aspects of virus biology can be understood in biomechanical terms at the nanoscale. A detailed knowledge of the relationship in virus capsids between small structural changes caused by single point mutations, and changes in mechanical properties may provide further physics-based insights into virus function; it may also facilitate the engineering of viral nanoparticles with improved mechanical behavior. Here we used the minute virus of mice to undertake a systematic experimental study on the contribution to capsid stiffness of amino acid side chains at interprotein interfaces and the specific noncovalent interactions they establish. Selected side chains were individually truncated by introducing point mutations to alanine, and the effects on local and global capsid stiffness were determined using atomic force microscopy. The results revealed that, in the natural virus capsid, multiple, mostly hydrophobic side chains buried along the interfaces between subunits preserve a comparatively low stiffness of most (S2 and S3) regions. Virtually no point mutation tested substantially reduced stiffness, while most mutations increased stiffness of S2/S3 regions. This stiffening was invariably associated to reduced virus yields during cell infection. The experimental evidence suggests that a comparatively low stiffness at S3/S2 capsid regions may have been biologically selected because it facilitates capsid assembly, increasing infectious virus yields. This study demonstrated also that knowledge of individual amino acid side chains and biological pressures that determine the physical behavior of a protein nanoparticle may be used for engineering its mechanical properties.
\end{abstract}

KEYWORDS: nanoparticle. virus. capsid. molecular structure and interactions. mechanical properties. stiffness. protein engineering. 
Viruses provide excellent model systems for physics-based studies of biological nanomachines. $^{1-15}$ The relationship between the atomic structure of virus particles and their response to mechanical forces is being explored using both computational and experimental approaches. In particular, coarse grained (CG) molecular dynamics (MD) ${ }^{11,12,16-21}$ combined with atomic force microscopy (AFM) $)^{6,9,10,13}$ has proved invaluable to link macroscopic mechanics with general structural aspects of different natural virus capsids. These and other studies are revealing a basic dependence of global mechanical properties on fundamental architectural similarities and differences between the capsids of different virus species. ${ }^{16-20}$

A complementary approach to study structure-mechanics relationships in viruses, and their possible biological relevance, is to compare any differences in mechanical behavior and biological function when single point mutations are introduced in the capsid of a same virus species, ${ }^{21-26}$ AFM has been found particularly suitable for such mutational analysis. ${ }^{22-26}$ There is strong biological interest in these studies because: i) viruses escape host defenses, adapt and evolve in nature in large part through the progressive accumulation of point mutations. ii) mechanical analysis by AFM of a limited number of point mutations in a same viral particle revealed that even single amino acid substitutions causing very small, local structural differences may result in large variations in global mechanical stiffness; these effects have been observed for cowpea chlorotic mottle virus (CCMV), ${ }^{22}$ minute virus of mice (MVM) ${ }^{23-25}$ and human immunodeficiency virus (HIV). ${ }^{26}$ iii) recent studies using nonenveloped viruses (bacteriophages, ${ }^{27-29} \mathrm{MVM}^{24,25}$ adenovirus, ${ }^{30,31}$ ) or enveloped viruses (HIV, ${ }^{32,33}$ influenza virus, ${ }^{34,35}$ ), revealed that variations in virus particle stiffness in a same viral species may be biologically relevant. Understanding in detail the relationships between fixation of single point mutations in virus particles, variations in mechanical properties and related alterations in biological function could lead to the development of antiviral strategies based on alteration of virus mechanical behavior.

The same mechanical features of natural viruses and their capsids that may have evolved to enable or facilitate biological function could jeopardize many of the promising applications of virus nanoparticles (NPs) that the advent of nanoscience and nanotechnology has opened up. Virus-based NPs are being tested, for example, as nanocontainers for targeted drug delivery; contrast agents for medical imaging; nanobiosensors; light harvesting devices; or templates to obtain metallic NPs for producing catalysts, batteries, electronic circuits and memory devices. ${ }^{36-43}$ Unfortunately, natural virus particles may not withstand the harsh conditions and mechanical forces they could meet during production ${ }^{44}$ and/or use. In addition, the lower stiffness of many virus particles compared to other NPs may make them inadequate 
for applications where a precise distance between active components must be preserved (e.g., between donor and acceptor in a light-harvesting $\mathrm{NP}^{42}$ ). Also, biologically relevant conformational dynamism in virus particles ${ }^{45-47}$ has been linked to limited mechanical stiffness. ${ }^{20,24,25}$ In virus-based NPs intended for drug delivery, low stiffness may lead to leaching of confined cargo molecules through flexible, dynamic capsid openings ${ }^{48}$ before reaching the target cell. Thus, the suitability of virus-based NPs for different applications could depend on the acquisition of fundamental knowledge on the structural determinants of virus mechanics, in order to rationally engineer their mechanical strength and/or stiffness, for example through the introduction of appropriate point mutations.

$\mathrm{MVM}^{49}$ one of the smallest (25 $\mathrm{nm}$ in diameter) and structurally simplest viruses known, constitutes an excellent model to study the contribution of individual amino acid residues to virus mechanics, the mechanical effects of single point mutations and their biological consequences. The MVM virion ${ }^{50,51}$ (Fig. 1) is formed by a single-stranded (ss) DNA genome enclosed in a nonenveloped $\mathrm{T}=1$ icosahedral capsid made of 60 structurally equivalent protein subunits with identical sequence and fold, except for their $\mathrm{N}$-terminal $(\mathrm{Nt})$ segments. The capsid is self-assembled from 20 trimers of subunits that constitute stable building blocks (Fig. 1b), ${ }^{52-54}$ and the viral ssDNA is packaged into the preformed capsid. ${ }^{49,55}$ Short segments of the viral ssDNA are folded as "wedges" that become noncovalently linked to concavities at the capsid inner wall, close to the 2-fold and 3-fold symmetry axes (Fig. 1b). ${ }^{50,51} \mathrm{The} \mathrm{Nt}$ segments, longer in 10 (VP1) subunits than in the 50 other (VP2) subunits, are disordered, initially located inside the capsid, and do not contribute to capsid architecture, but carry signals important for infection. ${ }^{49,52-63}$ Pores (channels) at the capsid 5-fold axes ${ }^{50,51}$ (Fig. 1b) are involved in DNA encapsidation and release, and in controlled translocation of VP1 and VP2 Nt segments as required for viral infection. ${ }^{49,52-63}$

Our previous studies on MVM mechanics revealed that the capsid-bound ssDNA segments act like molecular buttresses that stiffen most regions of the virion and impair a heat-induced, virus-inactivating structural change. ${ }^{23,25}$ In turn, amino acid residues at the base of each capsid pore contribute to preserve a low stiffness around the pores and facilitate a conformational rearrangement associated to through-pore translocation of biological signals during the infectious cycle. ${ }^{24}$ Those results led to the conclusion that the anisotropic distribution of mechanical stiffness in the infectious MVM virion provides the biological advantage of increasing its thermal resistance without impairing the pore-associated conformational dynamism required for infection. ${ }^{24,25}$ 
Here we have undertaken a systematic mutational analysis of the MVM capsid to reveal the individual contributions to local and global mechanical stiffness of a virus capsid, and their relationship to virus infectivity, of specific amino acid side chains at capsid intersubunit interfaces. Individual side chains with different stereochemistry, located in different capsid structural elements and involved in different types of noncovalent interactions between capsid subunits were genetically removed (beyond $\mathrm{C} \beta$ ) by mutation to alanine, and the effects on local and global mechanical stiffness were experimentally determined by AFM. Fundamental biological and nanotechnological implications of the results are discussed.

\section{RESULTS}

Selection of amino acid side chains in the MVM capsid for structure-mechanics relationship analysis. Site-directed mutagenesis was used to individually mutate to alanine different amino acid side chains at the interfaces between trimeric capsid building blocks. In this way, a series of mutant capsids were obtained. In each of them a single, specific amino acid side chain (beyond $C \beta$ ) had been removed in each of the 60 capsid subunits, without introducing any other chemical group. Of the side chains that are individually dispensable for capsid assembly, ${ }^{64}$ we focused in those that participate mainly or exclusively in interactions between trimers, and are free of interactions between subunits within each trimer. The selection of individual side chains to be analyzed (Fig. 2 and Table 1) was further based on the following features:

Strength of intertrimer interactions. Different side chains involved in interactions of different estimated binding affinity ${ }^{64,65}$ were chosen (Table 1).

Directional versus non-directional intertrimer interactions. Side chains chosen sampled those whose contribution to intertrimer association includes: i) directional hydrogen bonds (class I in Table 1); or ii) non-directional interactions only, including coulombic interactions (class II) in the absence of hydrogen bonding, or van der Waals contacts only, including C-C contacts (class III).

Location in different positions and structural elements in the capsid. Removal of any side chain buried in a virus capsid, in addition to disrupting interactions, would originate a small cavity. Thus, the mechanical effect of removing a side chain could strongly depend on its spatial position in the viral particle and possible conformational rearrangements if a cavity was created. We focused in side chains scattered along the intertrimer interfaces, from 
residues close to the capsid S2 symmetry axis at the center of each interface, to others closer to the S5 axes at its edges (Fig. 2, cyan residues). Some chosen groups are located in exposed loops, while others are buried within the $\beta$-barrel that constitutes the scaffold of each capsid subunit. The side chain of residue I167 was included because, even though its removal may not disrupt any intertrimer contact, it would enlarge a small cavity (gap) at the intertrimer interface (Table 1).

\section{Contributions of individual side chains and their interactions at the intertrimer} interfaces to the mechanical stiffness of the MVM capsid. The above considerations led to the selection of 12 different interfacial residues (Fig. 2 and Table 1) to quantify the effect of their individual mutation to alanine on capsid stiffness. The engineered MVM capsids and the control (wild-type, wt) capsid were produced, self-assembled and purified. For each single capsid analyzed, AFM imaging in physiological buffer was carried out to reveal particle height and major topographic features (large spikes at S3 axes, dimples at S2 axes and cylinders surrounding the pores at S5 axes). These features served to assess the integrity and orientation of single capsids (Fig. 1c). At the resolution achieved (a few $\AA$ in height), no significant size or structural differences were detected for any mutant relative to the wt capsid (not shown).

Then, the elastic constants, $k$, of S2, S3 and S5 regions of those virus particles in the same buffer were determined by indentation with the AFM tip of adequately oriented single particles. A controlled approach fully validated in our previous studies on other aspects of MVM mechanics was used (see Methods). Mutant capsids N74A and I167A had already served as negative controls in a study with a different purpose, ${ }^{24}$ the $k$ values previously obtained for these 2 capsids are included in the comparisons for completeness. For each region in each of the 10 mutant capsids tested here, many valid force-distance curves were obtained using several individual particles (Figs. 3 and 4 and Table 2). The average $k$ values determined for each region of each mutant capsid analyzed were compared with the corresponding values obtained for the wt capsid, and the differences were statistically validated (Table 2).

Fig. 5a summarizes these results by showing the differences in $k$ values between each mutant and the wt capsid. Only 2 out of the 12 single mutants showed a $k$ value that was substantially different (arbitrary cutoff: $>16 \%$ higher or lower) from the wt at S5 regions (1 stiffer, 1 softer); 9 out of the 12 single mutants showed a $k$ value that was substantially different from the wt at $\mathrm{S} 2$ regions (all of them stiffer); and 9 out of 12 single mutants showed a $k$ value that was substantially different from the wt at S3 regions (8 stiffer, 1 softer). In 
several other cases, less substantial $(\leq 16 \%)$ but still statistically significant changes in stiffness were observed.

In addition to this general trend for stiffening of most (S2 and S3) capsid regions when different point mutations were introduced at the intertrimer interfaces, we observed widely different quantitative effects in the stiffening achieved (Fig. 5a) and its anisotropy when comparing capsid regions (Fig. 5b).

\section{Effects on capsid stiffness of removing chemical groups with different contributions} to binding affinity between capsid building blocks. We compared first the contribution to stiffness of side chains involved in multiple, presumably stronger noncovalent interactions between trimers with others involved only in very few, weaker interactions or no intersubunit interactions at all (Figs. 3, 4 and 5). As summarized in Fig. 5a, 7 out of 9 mutants in which multiple intertrimer interactions of different types (Table 1) were removed were stiffened at $\mathrm{S} 2$ regions ( $k$ values increased from $+16 \%$ to $+52 \%$ ), and 6 were also stiffened at S3 regions $(+18 \%$ to $+94 \%)$. Large increases in stiffness $(+22 \%$ to $+70 \%)$ were also observed in S2 and/or S3 regions for mutant capsids I84A and I167A in which, respectively, only one van der Waals contact, or no interaction at the interfaces, had been removed (Table 1). In the I167A capsid, the chemical group removed was also not involved in intratrimer or intrasubunit interactions. A closer examination of $k$ values determined in this study (Table 2) confirmed a general lack of correlation between the estimated effect of the mutation on trimer-trimer binding affinity, ${ }^{64,65}$ and the measured effect on stiffness at S2 and/or S3 regions.

\section{Effects on capsid stiffness of removing chemical groups involved in either hydrogen} bonds or nondirectional interactions between capsid building blocks. We compared next the changes in stiffness for 6 mutant capsids in which intersubunit hydrogen bonds had been removed (Table 1 and Figs. 3 and 5) versus (vs.) 5 others in which only nondirectional interactions had been disrupted (Table 1 and Figs. 4 and 5). As summarized in Fig. 5a, variations in $k$ for mutants missing some hydrogen bonds compared to the wt capsid ranged from $-16 \%$ to $+38 \%$ (average $+13 \%$ ) in $\mathrm{S} 2$ regions, $-19 \%$ to $+44 \%$ (average $+11 \%$ ) in S3 regions, and $-26 \%$ to $+33 \%$ (average $+3 \%$ ) in S5 regions. Thus, some of the hydrogen bondremoving mutations had no significant effect on stiffness, and most caused a moderate stiffening of S2 and/or S3 regions.

The only mutant capsid tested in which a coulombic interaction had been removed without eliminating any hydrogen bond (K241A), showed increased stiffness at S2 regions $(+54 \%)$ but not at S3 regions compared to the wt capsid (Fig. 5a). In the 4 mutant capsids tested in which only van der Waals contacts had been removed, variations in $k$ ranged from 
$+22 \%$ to $+52 \%$ (average $+43 \%$ ) in S2 regions, from $+32 \%$ to $+94 \%$ (average $+51 \%$ ) in S3 regions, and from $-16 \%$ to $+11 \%$ (average $-3 \%$ ) in S5 regions (Fig. 5a). Thus, mutations that remove non-directional interactions lead to a substantial stiffening of S2 and, in nearly all cases, S3 regions. We found no correlation between a reduction in estimated binding affinity (for example, by considering the number of $\mathrm{C}-\mathrm{C}$ interactions removed), and the degree of stiffening (compare Table 1 and Fig. 5a).

\section{Effects on capsid stiffness of removing chemical groups located in different} structural elements at the interfaces between capsid building blocks. Finally, we compared the stiffening effect of removing chemical groups with regard to their spatial position and structural context in the capsid. The results obtained with the 12 mutant capsids (Fig. 5a) allowed their neat differentiation into two classes, according to their measured stiffness at S2 regions (i.e., around the centers of each trimer-trimer interface) (Fig. 6): i) five class $\mathrm{R}$ mutant capsids, which showed larger increases in stiffness $(+38 \%$ to $+54 \%$, average $+49 \%$ ) relative to the wt capsid; ii) seven class E mutant capsids, which showed no or only modest increases in stiffness $(-16 \%$ to $+25 \%$, average $+12 \%)$.

Mapping the mutated residues belonging to either class ( $\mathrm{R}$ or $\mathrm{E}$ ) in the capsid revealed clear structural differences between classes (Fig. 6 and Video S1 in Supporting Information). The chemical groups removed in class R mutants (red residues in Fig. 6 and Video S1) are generally located along the line that define the elongated core of each intertrimer interface. These groups belong to amino acid residues located in secondary structure elements buried in each capsid subunit (in one case, in the capsid inner wall), and most of them have a strong hydrophobic component. In contrast, the chemical groups removed in class E mutants (cyan residues in Fig. 6 and Video S1) are generally located in loops of each subunit at the capsid outer surface. These exposed loops are not tightly associated with the capsid protein subunit they belong to, but are extended on neighboring subunits with which they establish some superficial contacts only. In addition, every chemical group removed in class E mutants (except the I167 side chain) is fundamentally polar in nature and rather accessible to solvent. I167 is non-polar but establishes no intertrimer interactions and is partially exposed to solvent in the protruding $\beta$-cylinder that surrounds each capsid pore (Fig. 2).

Removal of most class $\mathrm{R}$ or E groups did stiffen also S3 regions at the centers of the trimers, despite these regions are farthest from the mutated residues and the intertrimer interfaces. Differences in stiffening were observed also at S3 regions when either class R or E groups were removed ( $+37 \%$ vs. $+23 \%$ respective averages), although these differences were less extreme than those observed at S2 regions (Fig. 5a). Removal of either class R or E 
groups led to generally insignificant effects on stiffness at S5 regions at the vertices between intertrimer interfaces, i.e., the pore regions ( $+2 \% v$ s. $-4 \%$ respective averages; Fig. 5a). To sum up, the greater stiffening effect of removing class $\mathrm{R}$ groups does not occur only at the vicinity of the removed group, but is a rather global phenomenon affecting most (S2 and S3) though not all (S5) regions in the capsid.

Effect on capsid stiffness of combining mutations at the interfaces between capsid building blocks. We asked then if the stiffening effects of individual mutations could be combined to engineer even stiffer viral capsids. We constructed 3 multiple mutants, each containing 2 or 3 stiffening mutations. Two of these three combinations of mutations (R83A/S550A and R83A/H72A/S550A) drastically impaired capsid assembly and could not be tested. The triple mutant capsid I84A/I207A/N554A did assemble, and proved to be one of the stiffest viral capsids tested to date (except at the pore regions), with $k_{\mathrm{S} 2}=1.05 \mathrm{~N} / \mathrm{m}, k_{\mathrm{S} 3}$ $1.19 \mathrm{~N} / \mathrm{m}$ and $k_{\mathrm{S} 5}=0.72 \mathrm{~N} / \mathrm{m}$ (Table 2). However, it was not much stiffer than the single mutant I207A (Table 2 and Fig. 5a). Thus, the mechanical effect of combining mutations that are quite distant from each other at the capsid intertrimer interfaces (Fig. 2) is not necessarily additive.

Effects on capsid stiffness of mutation L143V that increases the size of a cavity in the MVM capsid. In the Discussion, the effects on capsid stiffness of interfacial mutations (this study) are compared with those of other mutations analyzed in previous work on other aspects of virus mechanics. In order to provide a more complete comparison, the effect of mutation L143V was also analyzed here. L143V reduces the size of an amino acid side chain in the capsid, like the interfacial mutations tested here, but this particular residue delimits a conspicuous cavity within each capsid protein subunit (white circles in Fig. 1b). AFM analysis yielded the following elastic constants for L143V: $k_{\mathrm{S} 2}=1.19 \pm 0.27$ (59 Fz; 6 particles); $k_{\mathrm{S} 3}=0.96 \pm 0.23$ ( $76 \mathrm{Fz} ; 10$ particles); $k_{\mathrm{S} 5}=0.59 \pm 0.10$ (57 Fz; 6 particles). Thus, removal of just one methyl group delimiting a capsid cavity in mutant L143V had no effect on S5 stiffness, but led to substantial stiffening of S2 and S3 regions (by $+116 \%$ and $+78 \%$, respectively).

\section{Effects on virus infectivity and biological fitness of capsid-stiffening mutations} scattered along interfaces between subunits. Results described above showed that multiple amino acid side chains scattered along the interfaces between trimeric capsid building blocks contribute to maintain the MVM capsid in a state of comparatively low mechanical stiffness, as individual removal of the vast majority of these groups led to a general stiffening of S2/S3 capsid regions. We asked whether this mutation-induced stiffening of the S2/S3 regions in the MVM capsid could be associated to some detrimental effect for virus survival. 
Firstly, infectious virus titers in cell culture and capsid stiffness were compared for the wt and mutant virus variants individually carrying 10 of the interfacial mutations analyzed (Table 1). The 7 virus mutants whose capsid is stiffer than the wt capsid at both S2 and S3 regions, were significantly less infectious than the wt virus. The 3 mutants whose capsids are not stiffer at S3 regions (including K204A and K241A that were stiffer than the wt at S2 regions and N74A which was not) showed normal infectivity.

Secondly, ex vivo competition experiments were carried out to determine whether the 2 virions carrying mutations that stiffened the capsid S2 regions but not S3 regions showed a different biological fitness compared to the wt virion. Each of those mutant virions were mixed with the wt virion, and allowed to compete for multiplication in cultured cells in two duplicate, independent experiments as described in Methods. ${ }^{66}$ In both experiments, mutant K204A was readily outcompeted by the wt virus. Even when the former was in a large $(>20-$ fold) excess relative to the latter, the wt virus reached dominance in only 3-5 cycles of infection. In contrast, mutant K241A outcompeted the wt virus and became fully dominant after 1-2 cycles of infection in both experiments. Sequencing indicated that no additional mutations had been fixed in the capsid protein of this mutant during the serial infections.

Sequence comparisons of many viral strains of 12 parvovirus species evolutionarily closest to MVM (according to sequence alignment of their VP2 capsid protein) was carried out. All 10 interfacial mutations to alanine that stiffened the S3 and/or S2 regions were not observed to occur in nature (the only exception being alanine at position 207 in 5 viral species).

The above results together led us to tentatively propose that intrinsic mechanical stiffening of S3 regions (and may be also S2 regions) in the capsid could lead to impaired infectivity of the virus. It could be argued that, in addition to stiffening the capsid, the tested mutations could also have some stiffness-unrelated effect in some stage of the viral cycle that could be the actual cause for reduced infectivity. To provide further experimental support for a true connection between stiffened S3 and/or S2 regions and reduced MVM infectivity, we challenged this tentative hypothesis by using an entirely different set of mutations as follows.

In a previous, unrelated study by our group,${ }^{67}$ we had shown that single mutations of certain amino acid residues in the MVM capsid, different from those analyzed above, also impaired virus infectivity. These residues were scattered not along the interfaces between trimers, but instead at the interfaces between the individual capsid proteins that form each trimer. We considered that, if the association between stiffened S3 (and/or S2) capsid regions and reduced infectivity (observed so far by analyzing mutations at intertrimer interfaces) was 
fortuitous, the probability that every infectivity-reducing mutation at the different, intratrimer interfaces caused a similar capsid-stiffening effect was remote. Thus, we selected for mechanics-infectivity analysis a representative sample of these mutations (V86A, W283A, Q291A, S326A) ${ }^{67}$, and determined the elastic constants of the corresponding mutant capsids. All 4 mutations significantly stiffened S3 regions (Table 3). All 4 of them stiffened also S2 regions (Table 3; for one mutant more data would be required to statistically validate the observed difference in S2 stiffness). To sum up, the set of mutations at intratrimer interfaces behaved like the series of mutations at the intertrimer interfaces, and as predicted by the hypothesis of a true linkage between increased capsid stiffness at S3 (and S2) capsid regions and impaired virus infectivity.

\section{A mechanism to link stiffening of $\mathbf{S 3 / S 2}$ regions in the capsid and reduced virus}

infectivity. To provide further evidence for a true linkage between increased stiffness of S3 (and/or S2) capsid regions and reduced virus infectivity, we attempted to identify which specific process in the viral cycle could be impaired when these capsid regions are stiffened.

We considered DNA packaging first. As DNA-binding sites in the capsid overlap with S2/S3 regions ${ }^{50}$ (Fig. 1b), stiffening these regions could impair the conformational adjustment (induced-fit) between these sites and their ligand DNA segments, reducing affinity; in turn, this lower affinity could facilitate the extracellular release of the DNA through capsid pores, ${ }^{68}$ reducing the yield of infectious virions. We tested this possibility by using a representative sample of S2/S3-stiffening mutations (F55A, I84A, I167A, I207A; Table 2). It had been previously shown that controlled heating of MVM virions to $60-70^{\circ} \mathrm{C}$ leads to externalization of the viral DNA without capsid dissociation. ${ }^{68}$ Thus, we subjected those four mutants and wt virions to controlled heating to $65^{\circ} \mathrm{C}$, and compared their normalized residual infectious titers. None of the mutant virions was inactivated faster than the wt virion (Fig. S1), which suggests that S2/S3 stiffening may not impair MVM infectivity by facilitating the untimely release of the genome.

We then considered capsid self-assembly. MVM capsid assembly is known to require a conformational rearrangement of the trimeric building blocks. ${ }^{54}$ Stiffening the trimers and the capsid being formed (as reflected in stiffening the $\mathrm{S} 3$ region at the center of each trimer, and also S2 regions at the intertrimer interfaces) could impair the required conformational rearrangement between of interacting trimers partners. We tested this possibility by choosing again the same representative sample of S2/S3-stiffening mutations (F55A, I84A, I167A, I207A; Table 2), and comparing the assembly efficiency of each mutant capsid relative to wt capsid by in situ immunofluorescence analysis as described in Methods. All four capsid- 
stiffening single mutations tested did impair capsid assembly (Fig. S2). Consistent with this result, two triple mutants carrying several stiffening mutations were completely unable to assemble (see above). These results indicate that a limited stiffness of capsid subunits and of the capsid being formed by addition of trimers ${ }^{69}$ may be important for achieving a better assembly efficiency. Identification of a step (capsid assembly) in the MVM cycle that is invariably impaired by mutations eapsid stiffening at S3/S2 capsid regions provides further support for a true linkage between increased stiffness of these capsid regions and reduced virus infectivity.

\section{DISCUSSION}

The results of this study revealed that most (S2 and S3) regions in the capsid of the natural infectious MVM virus are kept in a state of comparatively low mechanical stiffness $(k$ value) by the action of evolutionarily highly conserved, mainly hydrophobic amino acid side chains buried along intersubunit interfaces (Fig. 6 and Video S1). We discuss here three aspects related to this finding: i) from a structural point of view, a connection with the results of some previous computational studies on capsid structure-mechanics relationships, and a possible mechanism by which different chemical groups and interactions may limit the stiffness of a quasi-spherical, hollow protein NP; ii) from a biological point of view, a possible selective advantage for a virus to limit the stiffness of some capsid regions; iii) from a nanotechnological point of view, some implications for engineering virus-derived NPs with improved mechanical properties.

Contributions to capsid mechanical stiffness of individual amino acid side chains at protein-protein interfaces. CG MD simulations have been used previously to investigate, for different virus species, the relationship between capsid structure and mechanical behavior. ${ }^{11,12,16-21}$ For example, Roos et al ${ }^{16}$ used 15 beads to represent each protein subunit in the hepatitis B virus capsid, and the virtual subunits were connected by 3 bonds based on Lennard-Jones and Coulomb potentials. Cieplak and Robbins ${ }^{17}$ and Kononova et al.$^{18}$ studied the CCMV capsid, and Cieplak and Robbins ${ }^{17,19}$ also compared the capsids of different virus species. In these three studies, each capsid residue was represented by a single interaction center at the position of $\mathrm{C} \alpha$ that interacted with other neighboring centers through a LennardJones potential that considered the actual distance between interaction centers. Those simulations provided good matches for experimental results on global capsid stiffness and 
other mechanical properties of those capsids. Also, $\mathrm{CG}$ MD simulations of mutant $\mathrm{K}_{4} 2 \mathrm{R}^{70}$ and wt CCMV capsids were qualitatively consistent with the experimentally observed global stiffenning caused by this mutation. ${ }^{21}$ All of these studies indicate that the global mechanical behavior of a virus capsid can be approximated by CG models based on actual C $\alpha$ positions in the capsid structure, without explicitly contemplating the different chemical nature of the amino acid side chains and the diverse nature of the actual interactions they establish. ${ }^{16-19}$

The results of the present experimental, systematic analysis on a mechanical effect of point mutations in a virus capsid are consistent with some conclusions of the CG MD simulations summarized above: in our study, the magnitude of the global mechanical effect (stiffening) of removing individual side chains in the capsid of a same virus species did not correlate with the type(s) of intersubunit electrostatic interactions removed; rather, it was dependent on the position in the capsid of the residues whose side chains were truncated.

In addition, the present study also revealed that: i) in many cases specific, local variations in capsid stiffness due to single point mutations occurred close to the mutated residue and/or at distant capsid regions; ii) variations in capsid stiffness were in many cases anisotropic, with some capsid regions but not others becoming much stiffer; iii) the more stiffened regions were not necessarily the ones closer to the mutation; iv) larger variations in stiffness (at S2 and/or S3 regions) were generally observed when the removed side chains were essentially buried at the intersubunit interfaces, involved in hydrophobic contacts and located in secondary structure elements within each subunit; small or non-significant variations in stiffness occurred by removing side chains that are fundamentally polar in nature, generally located in capsid surface loops and rather accessible to solvent. In a previous study with a different aim we eventually observed that large increases in MVM capsid stiffness (including stiffening of $\mathrm{S} 2$ and/or S3 regions), occurred also when other buried side chains, either located at the base of $\beta$-cylinders that delimit the S5 capsid pores, or around capsid cavities, were removed. ${ }^{24}$

Taking all these observations into account, we suggest the possibility that many buried amino acid side chains in the natural (wt) MVM capsid (Fig. 7), could act as either "spacers" or "tensors" that would prevent the capsid from collapsing into a more compact, mechanically more rigid state. Strong experimental support for this tentative hypothesis comes by the recently solved crystal structure of one stiffened mutant capsid (N.Verdaguer, personal communication): global stiffening of the MVM capsid caused by removal of a side chain at the base of the capsid pores ${ }^{24}$ has been traced to a subtle general compaction of the capsid structure that involves quite small but significant alterations in the position of many atoms, including the $\mathrm{C} \alpha$ atoms of many residues, many of them located far away from the mutation 
site. It remains to be seen if capsid stiffening by other point mutations, the ones analyzed in the present study, could be traced to a similar capsid compaction.

The experimental stiffness values determined here for many MVM capsid mutants that involve amino acid residues of a same virus capsid provide a large number of experimental constraints to be considered for future MD simulations on the quantitative mechanical effects of biologically relevant point mutations in a viral capsid. In particular, once the atomic coordinates of these point mutant capsids are determined and available, it would be very interesting to explore by $\mathrm{CG}$ MD whether the differences in $\mathrm{C} \alpha$ positions between wt and mutants of this large series of mutants are enough to recapitulate the observed stiffening effects caused by point mutations.

A biological role limiting mechanical stiffness of $S 2$ and $S 3$ regions in the MVM capsid? Our studies on different aspects of MVM mechanics now allows a comparison of stiffness of capsids belonging to two natural (wt) strains (p and i) of MVM and as many as 32 mutants carrying single point mutations at capsid intersubunit interfaces, or around capsid pores or cavities (Fig. 7). Both natural capsids, despite they differ in 13 amino acids per subunit, showed nearly identical elastic constants that were lower than those of most point mutants tested. Moreover, only one region in one mutant (S5 in S550A) yielded an elastic constant that was substantially lower than that of the wt capsid. If a lower capsid stiffness had no biologically adaptive value, the probability of the wt capsids ( $p$ and i) being less stiff than the vast majority of point mutants tested would be quite small. It was also revealing that the capsid-stiffening mutations are virtually absent in sequenced natural populations of MVM and evolutionarily related parvoviruses (this study and unpublished observations). It looks as if the naturally evolved MVM capsid is kept in a local minimum of mechanical stiffness in the sequence space, and only point mutations that do not significantly increase capsid stiffness are tolerated. A number of additional experimental results obtained in this study (discussed next) indicate that this comparatively low capsid stiffness of the natural capsid may indeed be biologically required by the virus.

First, of the 12 tested point mutations of residues located at intertrimer interfaces but not at the base of the pores, only R83A (not far from the pores) stiffened the pore (S5) regions and was essentially deleterious. This observation confirm our previous results that stiffness at S5 regions is locally (and not globally) controlled, and that a relatively low stiffness around the capsid pores is strictly required for MVM infectivity. ${ }^{24}$ 
In addition, evidence obtained in the present study led us to hypothesize that a comparatively low stiffness at S3 (and perhaps also S2) regions in the capsid may provide a selective advantage to MVM by enhancing viral infectivity:

i) Mutant N74A, whose capsid was not stiffer than the wt at S2 and S3 regions, was normally infectious. In contrast, all 19 tested viruses with mutations that stiffened the capsid at $\mathrm{S} 3$ regions showed significantly reduced infectivity. These mutants included 10 viruses with point mutations at intertrimer interfaces and 4 with mutations at intratrimer interfaces (this study), and 5 other viruses ${ }^{71}$ with infectivity-reducing mutations around cavities inside the capsid wall (L143V analyzed here, and 4 others ${ }^{24}$ ). The correlation between stiffening of $\mathrm{S} 3$ regions in the capsid and reduced infectivity showed no exception. The association of reduced infectivity with S2 stiffening was less clear. In particular, two mutant viruses whose capsids were stiffened at S2 but not S3 regions were as infectious as the wt virus. While one of these (K204A) had a strongly reduced biological fitness, the other (K241A) was fitter than the natural virus in cell culture. However, the K241A mutation was never found among MVM and related parvoviruses in nature, which suggests a biological disadvantage. We had determined biological fitness in cell culture conditions; thus, it cannot be excluded that K241A fitness may be impaired in nature.

ii) After we formulated the hypothesis of a linkage between stiffened S3 (and may be S2) capsid regions and reduced MVM infectivity, we challenged it by using an independent set of mutations that were located within each trimer; these mutations were entirely unrelated to the mutations at the intertrimer interfaces that had been used to formulate the hypothesis. All mutations used to challenge the hypothesis both reduced virus infectivity and stiffened S3 and $\mathrm{S} 2$ regions as predicted.

iii) Further experimental evidence for a true connection between increased stiffness of S3/S2 capsid regions and reduced virus infectivity was provided by showing that, when tested, capsid S3/S2-stiffening, virus infectivity-reducing mutations invariably impaired a particular stage in the infectious cycle, capsid assembly. This result is consistent with the established fact that a conformational rearrangement of the trimeric building blocks is required for MVM capsid assembly. ${ }^{54,67}$ Stiffening the trimers and the capsid being assembled from them, ${ }^{69}$ as reflected in stiffening the $\mathrm{S} 3$ region at the center of each trimer and $\mathrm{S} 2$ regions at their borders, may likely impair such conformational rearrangement.

In dealing with complex biological systems including viruses, it is frequently very difficult to firmly establish cause-effect relationships. The ensemble of experimental results discussed above provides, in our view, a strong case for a true linkage between increased 
capsid stiffness around S3 regions and reduced virus infectivity. However, this hypothesis should be further reinforced in future studies by: i) simultaneously reversing the effects on S3 capsid stiffening, impaired capsid assembly and impaired virus infectivity; ii) exploring the biological effects of the stiffening mutations in more detail; and iii) undertaking MD simulations in order to firmly establish the structural basis that may connect changes in mechanical stiffness due to single point mutations and alteration of biologically relevant conformational changes during capsid assembly.

At this point it may be emphasized that, in the laboratory, we were able to increase the resistance of the MVM virion against heat-induced inactivation of its infectivity by introducing point mutations that stiffened $\mathrm{S} 2 / \mathrm{S} 3$ regions in the capsid. ${ }^{25}$ In contrast, in nature MVM appears to have increased virion resistance to heat-induced inactivation by a much more complex route: through fixation of enough mutations that created complex DNA binding sites at the capsid inner wall, thus stiffening S2/S3 regions in the DNA-filled virion but not in the empty capsid. We tentatively suggest that this convoluted solution may be a compromise in the face of conflicting selective pressures: on one hand, the need to preserve a low stiffness of the trimers and the capsid while the latter is being assembled; on other hand, the need to increase the stiffness of S2/S3 regions in the infectious virion, once the DNA has been packaged into the capsid, to increase virion thermostability.

A hypothetical empirical model that combines current structural and biological knowledge on MVM with the results of our studies on the mechanical behavior of the empty capsid ( ${ }^{24}$ and this study) and DNA-filled virion ${ }^{25}$ is the following: i) efficient assembly of the empty capsid requires a limited stiffness of the trimeric subunits and of the capsid being assembled (reflected in limited stiffness of S3 and S2 regions) (this study) to allow a conformational rearrangement of the trimer ${ }^{54}$ that improves recognition between trimers that are being added to the growing capsid during assembly; ${ }^{69}$ ii) later packaging of the viral DNA and binding of DNA segments to the capsid inner wall stiffens (mainly) the S2 regions in the DNA-filled virion), and thus makes the virion more resistant against heat-induced inactivation of its infectious capacity, ${ }^{25}$ by impairing the untimely release of the DNA ${ }^{68}$ through capsid openings; iii) absence of DNA binding at S5 regions around the pores and a number of conserved amino acid residues in the capsid preserve a relatively low stiffness around the pores, which allows the conformational rearrangement associated to through-pore translocation of peptide signals required for viral infectivity. ${ }^{24}$

Protein engineering of the MVM capsid provides mechanically tuned viral NPs. Systematic investigation of the structural determinants of viral capsid stiffness as carried out 
in this study has provided relevant clues for tuning by protein engineering the stiffness of virus-based and protein-based NPs. Analogous to the situation with other materials (e.g., rubber or plastic), our results suggest that variations in stiffness that can be introduced in a protein-based material through modest molecular alterations are subjected to intrinsic limitations. As discussed above, the wt MVM capsid appears to have been "engineered" by nature to preserve a state of comparatively low stiffness; thus, the rational design of MVM capsids of even lower stiffness by genetically introducing point mutations could be a difficult proposition. In contrast, the engineering of capsids that are greatly stiffened was extremely successful. An upper limit of stiffness may, however, have been reached for these viral NPs, as a combination of two or three different stiffening modifications either barely increased the (already very large) stiffening achieved by one of those modifications alone, or prevented capsid assembly.

Despite these (physically or biologically) imposed limitations, the present study did lead to a series of mechanically stiffened viral NPs offering multiple choices regarding their degree of global and/or local stiffness ( $k$ values ranging from $\sim 0.45$ to $\sim 1.20 \mathrm{~N} / \mathrm{m}$ ) and mechanical anisotropy (Fig. 5). Differences in size, geometry and architecture of the different point mutant capsids and wt capsid of a same virus species may be inexistent or very small (e.g. ${ }^{70}$ and, for MVM, N. Verdaguer, personal communication). Thus, for the mutants and wt capsids analyzed here, the differences between the corresponding values of the Young's modulus of elasticity ( $1 \mathrm{GPa}$ to $2.7 \mathrm{GPa}$ if calculated as described in $^{72,73}$ ) may parallel the differences in elastic constant values that were experimentally obtained.

\section{CONCLUSIONS}

This study undertook a systematic experimental dissection of the contribution in a natural virus capsid of different amino acid side chains at intersubunit interfaces to local and global mechanical stiffness. Many side chains, most of them hydrophobic, located in secondary structure elements, and buried along the interfaces between capsid subunits, contributed to maintain most (S2/S3) regions of the viral capsid in a state of low stiffness compared to mutant capsids in which those side chains had been individually truncated. A low stiffness of S3 (and may be S2) regions in the MVM capsid appears to be required for high capsid assembly efficiency and viral infectivity and may have been biologically selected. From an applied perspective, a family of engineered virus-based NPs was obtained from which one could choose the particle with the most adequate combination of global and local stiffness. 
Successful improvement of mechanical properties of protein-based NPs may require a detailed knowledge of atomic groups, and even biological pressures, that determined their natural mechanical behavior at the nanoscale.

\section{METHODS}

\section{Recombinant plasmids and construction and production of mutant empty capsids} and virions of MVM. Site-directed mutagenesis using the QuikChange kit (Stratagene) was used on plasmid pFB1-VP2 ${ }^{74}$ to introduce mutations in the VP2 gene of MVM. The presence of the mutations was confirmed by automated DNA sequencing. Then, the baculovirus expression system (Invitrogen) and mutant pFB1-VP2 as donor plasmids were used to construct the corresponding mutant BM-VP2 bacmids. ${ }^{74} \mathrm{BM}-\mathrm{VP} 2$ bacmids containing either the wt VP2 gene or different mutants ${ }^{64}$ were used for the production in H5 insect cells of empty capsids made of VP2 subunits exclusively. ${ }^{64}$ Plasmid pSVtk-VP1/2 in which the appropriate mutations had been introduced ${ }^{24}$ were used for the production in NB324K cells of mutant empty capsids ${ }^{52,57}$ (N74A, I167A, and the capsids carrying intratrimer mutations (L143V and the mutants in Figs. S1 and S2). The crystal structures of both types of capsids (VP2 and VP1/VP2) are indistinguishable in both structure and stiffness (the Nts are disordered and do not contribute to capsid architecture) $\left({ }^{51}\right.$, this study and unpublished data). Mutant virions were originally obtained by transfection of NB324K cells ${ }^{64}$ as described previously, ${ }^{62}$ using infectious clones derived from the original MVMp infectious clone, ${ }^{75}$ and amplified by infection of cultured cells.

Purification of MVM capsids and virions. It was carried out essentially as previously described. ${ }^{52,57,64}$ Purity and quality of the preparations was primarily assessed by electron microscopy.

Virus infectivity, thermal inactivation and competition assays. Virus infectious titers were determined in duplicate in standard plaque-formation assays and normalized as described. $^{24}$ To ensure comparable results, a control wt virus was included in each experiment.

Virus inactivation assays were carried out by incubating the same number of purified wt and mutant infectious virions $\left(\sim 2 \times 10^{5}\right.$ plaque-forming units) at $65^{\circ} \mathrm{C}$. Aliquots were taken at different incubation times (up to $2 \mathrm{~h}$ ), and remaining infectious titers were determined in duplicate as above. Two independent experiments were carried out. Titers were normalized relative to the original titer (before heating) and averaged. 
Virus competition experiments were carried out using a previously described procedure ${ }^{66}$ that we adapted for MVM. Briefly, NB324K cell monolayers were infected using equal titers of the competing viruses at a total multiplicity of infection of 0.5. Progeny viruses were harvested at $72 \mathrm{~h}$ post-infection, a fraction of the progeny virus population was used to infect fresh cell monolayers, and this procedure was repeated until five successive cycles of infection and harvest had been completed. For each pair of competing viruses, the experiment was repeated twice. The viral ssDNA from the populations obtained after different cycles was obtained, amplified by the polymerase chain reaction and sequenced. The approximate proportion of the competing viruses in the populations was estimated by densitometry of the chromatogram peaks corresponding to the nucleotide present in the mutant virus genome and the nucleotide present in the wt virus genome.

Immunofluorescence assays. The efficiency of assembly of mutant capsids relative to the wt was determined essentially as previously described. ${ }^{67}$ Briefly, aliquots of NB324K cells were electroporated in parallel using the same amounts of wt and mutant pSVtk-VP1/2, and grown in monolayers on coverslips for $48 \mathrm{~h}$. The amounts of both total capsid protein produced and assembled capsids were respectively determined by in situ immunofluorescence using specific primary antibodies. ${ }^{67}$ Two independent experiments were carried out for each mutant using both a negative control and wt-transfected cells as a positive control. Capsid/VPs ratios (relative assembly efficiency values) were obtained by counting fluorescent cells, and normalized relative to the ratio determined for the wt control. ${ }^{67}$

Atomic force microscopy. AFM hardware and software was from Nanotec Electrónica, S.L. AFM imaging and determination of the mechanical stiffness of individual wt or mutant MVM capsids was carried out essentially as previously described. ${ }^{23,72}$ Briefly, a drop of the sample $(20 \mu \mathrm{l})$ in PBS was deposited onto a hexamethyldisilazane (Sigma-Aldrich)-treated glass coverslip and left for $30 \mathrm{~min}$ at room temperature for capsid adsorption. AFM images were obtained in Jumping Mode ${ }^{76}$ using RC800PSA cantilevers (Olympus) with a nominal spring constant of $0.1 \mathrm{~N} / \mathrm{m}$ and keeping the applied force under $\sim 50 \mathrm{pN}$. Before starting each experiment, the actual spring constant of each cantilever used was calibrated using Sader's method. ${ }^{77}$ AFM images were processed using WSxM software. ${ }^{78}$ The stiffness of different regions in the MVM capsid was determined by indenting capsid particles of known orientation with a 5-fold, 3-fold or 2-fold symmetry axis close to the top of the particle at the indentation point. ${ }^{73}$ To keep within the range of a linear elastic response and avoid particle damage, only force-vs.-distance $(\mathrm{Fz})$ measurements that involved indentations between 0.5 $\mathrm{nm}$ and $2.0 \mathrm{~nm}$ were considered as previously justified. ${ }^{24}$ The local stiffness of each capsid 
region (around a 5-fold, 3-fold or a 2-fold axis) was determined by calculating the corresponding spring constant $k$, assuming that viral particle and AFM cantilever behave as two ideal springs in series. ${ }^{23,72,73}$

Statistical analysis of mechanical data. The $k$ values obtained for MVM particles followed a normal distribution described by a Gaussian fitting, as corroborated using normality tests (and as observed for other viral particles). The statistical significance of differences in $k$ values between mutant and parent capsids was assessed using OriginPro8 (OriginLab). As in previous studies ${ }^{24}$ to statistically validate that two $k$ are different, the twopopulation two-tailed Student t-test was used, with an alpha level of 0.05. Equal variance was not assumed, providing a more stringent test.

Sequence alignment, molecular graphics and structural analysis. Alignment of 64 parvoviral capsid protein sequences were carried out with the program Clustal. ${ }^{79}$ The programs WHAT IF, ${ }^{80} \mathrm{VMD}^{81}$ and $\mathrm{Pymol}^{82}$ and the atomic coordinates for the crystallographic structure of the MVMp capsid ${ }^{51}$ (1Z14) were used for structure inspection, analysis of interactions, design of mutations, interpretation of results and/or molecular graphics display.

Acknowledgments. We gratefully acknowledge J.M. Almendral for scientific support and providing biological material, N. Verdaguer and P.J. de Pablo for scientific discussion and D. Reguera and R. Pérez for discussion and critical reading of the manuscript. P.J.P.C. and A.V. were the respective recipients of a FPI fellowship and a post-doctoral contract from the Spanish Ministerio de Economía y Competitividad (MINECO), and M.M. was granted a FPI fellowship from Universidad Autónoma de Madrid. This work was funded by grants BIO2012-37649 and BIO2015-69928-R (MINECO/FEDER) to M.G.M., and an institutional grant from Fundación Ramón Areces to the Centro de Biología Molecular. M.G.M. is an associate member of the Institute for Biocomputation and Physics of Complex Systems, Zaragoza, Spain.

Supporting Information Available: Video S1 (an animated view of Fig. 6) and Figs. S1 and $\mathrm{S} 2$. This material is available free of charge via the Internet at http://pubs.acs.org. 


\section{REFERENCES}

1.Smith, D.E.; Tans, S.J.; Smith, S.B.; Grimes, S.; Anderson, D.L.; Bustamante, C. The Bacteriophage $\$ 29$ Portal Motor Can Package DNA Against a Large Internal Force. Nature 2001, 413, 748-752.

2.Bustamante, C.; Bryant, Z.; Smith, S.B. Ten Years of Tension: Single-Molecule DNA Mechanics. Nature 2003, 421, 423-427.

3.Perlmutter, J.D.; Hagan, M.F. Mechanisms of Virus Assembly. Annu. Rev. Phys. Chem. 2004, 66, 217-239.

4.Knobler, C.M.; Gelbart, W.M. Physical Chemistry of DNA Viruses. Annu. Rev. Biochem. 2009, 60, 367-383.

5.Gelbart, W.M.; Knobler, C.M. Pressurized Viruses. Science 2009, 323, 11216-11221.

6.Roos, W.; Bruinsma, R.; Wuite, G. Physical Virology. Nature Phys. 2010, 6, 733-743.

7.Stockley, P.G.; Twarock, R. (Eds.). Emerging Topics in Physical Virology; Imperial College Press: London, UK, 2010.

8.Rossman, M.G.; Rao, V.B. (Eds.). Viral Molecular Machines. Advances in Experimental Medicine and Biology vol. 726; Springer: New York, NY, 2012.

9.Mateu, M.G. Mechanical Properties of Viruses Analyzed by Atomic Force Microscopy: a Virological Perspective. Virus Res. 2012, 168, 1-22.

10.de Pablo, P.J.; Mateu, M.G. Mechanical Properties of Viruses. In Structure and Physics of Viruses, M.G. Mateu, Ed.; Springer: Dordrecht, The Netherlands, 2013; pp. 519-551.

11.Luque, A.; Reguera, D. Theoretical Studies on Assembly, Physical Stability and Dynamics of Viruses. In Structure and Physics of Viruses, M.G. Mateu, Ed.; Springer: Dordrecht, The Netherlands, 2013; pp. 553-595.

12.May, E.R. Recent Developments in Molecular Simulation Approaches to Study Spherical Virus Capsids. Mol. Simul. 2014, 40, 878-888.

13.van Rosmalen, M.G.; Roos, W.H.; Wuite, G.J. Material Properties of Viral Nanocages Explored by Atomic Force Microscopy. Methods Mol. Biol. 2015, 1252, 115-137.

14.Greber, U.F. Virus and Host Mechanics Support Membrane Penetration and Cell Entry. J. Virol. 2016, 90, 3802-3805.

15.Hagan, M.F.; Zandi, R. Recent Advances in Coarse-Grained Modeling of Virus Assembly. Curr. Opin. Virol. 2016, 18, 36-43.

16.Roos, W.H.; Gibbons, M.M.; Arkhipov, A.; Uetrecht, C.; Watts, N.R.; Wingfield, P.T.; Steven, A.C.; Heck, A.J.R.; Schulten, K.; Klug, W.S.; Wuite, G.J.L. Squeezing Protein 
Shells: How Continuum Elastic Models, Molecular Dynamics Simulations, and Experiments Coalesce at the Nanoscale. Biophys. J. 2010, 99, 1175-1181.

17.Cieplak, M.; Robbins, M.O. Nanoindentation of Virus Capsids in a Molecular Model. $J$. Chem. Phys. 2010, 132, 015101.

18.Kononova, O.; Snijder, J.; Brasch, M.; Cornelissen, J.; Dima, R.I.; Marx, K.A.; Wuite,

G.J.L.; Roos, W.H.; Barsegov, V. Structural Transitions and Energy Landscape for Cowpea Chlorotic Mottle Virus Capsid Mechanics from Nanomanipulation in Vitro and in Silico. Biophys. J. 2013, 105, 1893-1903.

19.Cieplak, M.; Robbins, M.O. Nanoindentation of 35 Virus Capsids in a Molecular Model: Relating Mechanical Properties to Structure. PLoS One 2013, 6, e63640.

20.Kononova, O.; Snijder, J.; Kholodov, Y.; Marx, K.A.; Wuite, G.J.L.; Roos, W.H.; Barsegov, V. Fluctuating Nonlinear Spring Model of Mechanical Deformation of Biological Particles. PLoS Comp. Biol. 2016, 12, e1004729.

21. Globisch, C.; Krishnamani, V.; Deserno, M.; Peter, C. Optimization of an Elastic Network Augmented Coarse Grained Model to Study CCMV Capsid Deformation. PLoS One 2013, 8, e60582.

22.Michel, J.P.; Ivanovska, I.L.; Gibbons, M.M.; Klug, W.S.; Knobler, C.M.; Wuite, G.J.L.; Schmidt, C.F. Nanoindentation Studies of Full and Empty Viral Capsids and the Effects of Protein Mutations on Elasticity and Strength. Proc. Natl. Acad. Sci. USA 2006, 103, 61846189.

23.Carrasco, C.; Castellanos, M.; de Pablo, P.J.; Mateu, M.G. Manipulation of the Mechanical Properties of a Virus by Protein Engineering. Proc. Natl. Acad. Sci. USA 2008, 105, 41504155 .

24.Castellanos, M.; Pérez, R.; Carrasco, C.; Hernando-Pérez, M.; Gómez-Herrero, J.; de Pablo, P.J.; Mateu, M.G. Mechanical Elasticity as a Physical Signature of Conformational Dynamics in a Virus Particle. Proc. Natl. Acad.Sci. USA 2012, 109, 12028-12033.

25.Castellanos, M.; Carrillo, P.J.P.; Mateu, M.G. Quantitatively Probing Propensity for Structural Transitions in Engineered Virus Nanoparticles by Single-Molecule Mechanical Analysis. Nanoscale 2015, 19, 5654-5664.

26.Ramalho, R.; Rankovic, S.; Zhou, J.; Aiken, C.; Rousso, I. Analysis of the Mechanical Properties of Wild-Type and Hyperstable Mutants of the HIV-1 Capsid. Retrovirology 2016, $13,17$.

27.Ivanovska, I.L.; Wuite, G.; Jönsson, B.; Evilevitch, A. Internal DNA Pressure Modifies 
Stability of Wt Phage. Proc. Natl. Acad. Sci. USA 2007, 104, 9603-9608.

28.Roos, W.H.; Gertsman, I; May, E.R.; Brooks, C.L. 3rd; Johnson, J.E.; Wuite, G.J. Mechanics of Bacteriophage Maturation. Proc. Natl. Acad. Sci. USA 2012, 109, 23422347.

29.Hernando-Pérez, M.; Lambert, S.; Nakatani-Webster, E.; Catalano, C.E.; de Pablo, P.J. Cementing Proteins Provide Extra Mechanical Stabilization to Viral Cages. Nat. Commun. $20145,4520$.

30.Ortega-Esteban, A.; Condezo, G.N.; Pérez-Berná, A.J.; Chillón, M.; Flint, J.; Reguera, D.; San Martín, C.; de Pablo, P.J. Mechanics of Viral Chromatin Reveals the Pressurization of Human Adenovirus. ACS Nano 2015, 9, 10826-10833.

31.Ortega-Esteban, A.; Bodensiek, K.; San Martín, C.; Suomalainen, M.; Greber, U.F.; de Pablo, P.J.; Schaap, I.A.T. Fluorescence Tracking of Genome Release During Mechanical Unpacking of Single Viruses. ACS Nano 2015, 9, 10571-10579.

32.Kol, N.; Shi, Y.; Tsvitov, M.; Barlam, D.; Schneck, R.Z; Kay, M.S.; Rousso, I. A Stiffness Switch in Human Immunodeficiency Virus. Biophys. J. 2007, 92, 1777-1783.

33.Pang, H.B.; Hevroni, L.; Kol, N.; Eckert, D.M.; Tsvitov, M; Kay, M.S; Rousso, I. Virion Stiffness Regulates Immature HIV-1 Entry. Retrovirology 2013, 10, 4.

34.Li, S.; Sieben, C.; Ludwig, K.; Höfer, C.T., Chiantia, S.; Hermann, A.; Eghiaian, F.; Schaap. I.A.T. pH-Controlled Two-Step Uncoating of Influenza Virus. Biophys. J. 2014, $106,1447-1456$.

35.Greber, U.F. How Cells Tune Viral Mechanics-Insights from Biophysical Measurements of Influenza Virus. Biophys. J. 2014, 106, 2317-2321.

36.Douglas, T.; Young, M. Viruses: Making Friends with Old Foes. Science 2006, 312, 873875.

37.De la Escosura, A.; Nolte, R; Cornelissen, J. Viruses and Protein Cages as Nanoconainers and Nanoreactors. J. Mater. Chem. 2009, 19, 2274-2278.

38.Yildiz, I.; Shukia, S.; Steinmetz, N.F. Applications of Viral Nanoparticles in Medicine. Curr. Opin. Biotechnol. 2011, 22, 901-908.

39.Mateu, M.G. Virus Engineering: Functionalization and Stabilization. Prot. Eng. Des. Sel. 2011, 24, 53-63.

40.Bittner, A.M., Alonso, J.M., Górzny, M.L., Wege, C. Nanoscale Science and Technology with Plant Viruses and Bacteriophages. In Structure and Physics of Viruses, M.G. Mateu, Ed.; Springer: Dordrecht, The Netherlands 2013; pp. 667-702. 
41.Wen, A.M.; Rambhia, P.H.; French, R.H.; Steinmetz, N.F. Design Rules for Nanomedical Engineering: from Physical Virology to the Applications of Virus-Based Materials in Medicine. J.Biol.Phys. 2013, 39, 301-325.

42.Li, F.; Wang, Q. Fabrication of Nanoarchitectures Templated by Virus-Based Nanoparticles: Strategies and Applications. Small 2014, 10, 230-245.

43.Mateu, M.G. Assembly, Engineering and Applications of Virus-Based Protein Nanoparticles. In Protein-Based Engineered Nanostructures, A.L. Cortajarena, T.Z. Grove, Eds.; Springer: London, UK. 2016; pp. 83-120.

44.Powell, S.K.; Kaloss, M.A.; Pinkstaff, A.; McKee, R.; Burimski, I.; Pensiero, M.; Otto, E.;

Stemmer, W.P.C.; Soong, N.-W. Breeding of Retroviruses by DNA Shuffling for Improved Stability and Processing Yields. Nat. Biotechnol. 2000, 18, 1279-1282.

45.Johnson, J.E. Virus Particle Dynamics. Adv. Protein Chem. 2003, 64, 197-218.

46.Bothner, B.; Hilmer, J.K. Probing Viral Capsids in Solution. In Structural Virology, M.

Agbandje-McKenna, R. McKenna, Eds.; RSC Publishing: Cambridge, UK 2011; pp. 4161.

47.Mateu, M.G. Assembly, Stability and Dynamics of Virus Capsids. Arch. Biochem. Biophys. 2013, 532, 65-79.

48.Ren, Y.; Wong, S.M.; Lim, L.Y. In Vitro-Reassembled Plant Virus-Like Particles for Loading of Polyacids. J. Gen. Virol. 2006, 87, 2749-2754.

49.Cotmore, S.F.; Tattersall, P. Parvoviruses: Small Does Not Mean Simple. Annu. Rev. Virol. 2014, 1, 517-537.

50.Agbandje-McKenna, M.; Llamas-Saiz, A.L.; Wang, F.; Tattersall, P.; Rossmann, M.G. Functional Implications of the Structure of the Murine Parvovirus, Minute Virus of Mice. Structure 1998, 6, 1369-1381.

51.Kontou, M; Govindasamy, L.; Nam, H.J.; Bryant, N.; Llamas-Saiz, A.L.; Foces-Foces, C.;

Hernando, E.; Rubio, M.P.; McKenna, R.; Almendral, J.M.; Agbandje-McKenna, M.

Structural Determinants of Tissue Tropism and in Vivo Pathogenicity for the Parvovirus Minute Virus of Mice. J. Virol. 2005, 79, 10931-10943.

52.Lombardo, E.; Ramírez, J.C.; García, J.; Almendral, J.M. Complementary Roles of Multiple Nuclear Targeting Signals in the Capsid Proteins of the Parvovirus Minute Virus of Mice During Assembly and Onset of Infection. J. Virol. 2002, 76, 7049-7059.

53.Riolobos, L.; Reguera, J.; Mateu, M.G.; Almendral, J.M. Nuclear Transport of Trimeric Assembly Intermediates Exerts a Morphogenetic Control on the Icosahedral Parvovirus Capsid. J. Mol. Biol. 2006, 357, 1026-1038. 
54.Almendral, J.M. Assembly of Simple Icosahedral Viruses. In Structure and Physics of Viruses, M.G. Mateu, Ed.; Springer: Dordrecht, The Netherlands, 2013; pp. 307-328.

55.Plevka, P.; Hafenstein, S.; Li, L.; D’Abramo, A. Jr.; Cotmore, S.F.; Rossmann, M.G.;

Tattersall, P. Structure of a Packaging-Defective Mutant of Minute Virus of Mice Indicates That the Genome is Packaged Via a Pore At A 5-Fold Axis. J. Virol. 2011, 85, 4822-4827.

56.Cotmore, S.F.; D’Abramo, A.M. Jr.; Ticknor, C.M.; Tattersall, P. Controlled Conformational Transitions in the MVM Virion Expose the VP1 N-Terminus and Viral Genome without Particle Disassembly. Virology 1999, 254, 169-181.

57.Hernando, E.; Llamas-Saiz, A.L.; Foces-Foces, C.; McKenna, R.; Portman, I.; AgbandjeMcKenna, M.; Almendral, J.M. Biochemical and Physical Characterization of Parvovirus Minute Virus of Mice Virus-Like Particles. Virology 2000, 267, 299-309.

58.Maroto, B.; Valle, N.; Saffrich, R.; Almendral, J.M. Nuclear Export of the Nonenveloped Parvovirus Virion is Directed by an Unordered Protein Signal Exposed on the Capsid Surface. J. Virol. 2004, 78, 10685-10694.

59.Farr, G.A.; Cotmore, S.F.; Tattersall, P. VP2 Cleavage and the Leucine Ring at the Base of the Fivefold Cylinder Control pH-Dependent Externalization of Both the VP1 N-Terminus and the Genome of Minute Virus of Mice. J. Virol. 2006, 80, 161-171.

60.Valle, N.; Riolobos, L.; Almendral, J.M. Synthesis, Post-Translational Modification and Trafficking of the Parvovirus Structural Polypeptides. In Parvoviruses. J.R. Kerr, S.F. Cotmore, M.E. Bloom, R.M. Linden, C.R. Parrish, Eds. Edward Arnold: London, UK, 2006, pp. 291-304.

61.Cotmore, S.F.; Tattersall, P. Parvoviral Host Range and Cell Entry Mechanisms. Adv. Virus Res. 2007, 70, 183-232.

62.Sánchez-Martínez, C.; Grueso, E.; Carroll, M.; Rommelaere, J.; Almendral, J.M. Essential Role of the Unordered VP2 N-Terminal Domain of the Parvovirus MVM Capsid in Nuclear Assembly and Endosomal Enlargement of the Virion Fivefold Channel for Cell Entry. Virology 2012, 432, 45-56.

63.Cotmore, S.F.; Tattersall, P. Mutations at the Base of the Icosahedral Five-Fold Cylinders of Minute Virus of Mice Induce 3'-to-5'Genome Uncoating and Critically Impair Entry Functions. J. Virol. 2012, 86, 69-80.

64.Reguera, J.; Carreira, A.; Riolobos, L.; Almendral, J.M.; Mateu M.G. Role of Interfacial Amino Acid Residues in Assembly, Stability, and Conformation of a Spherical Virus Capsid. Proc. Natl. Acad. Sci. USA 2004, 101, 2724-2729. 
65.Carrillo-Tripp, M.; Shepherd, C.M.; Borelli, I.A.; Venkataraman, S.; Lander, G.; Natarajan, P.; Johnson, J.E.; Brooks, C.L. 3rd.; Reddy, V.S. VIPERdb2: an Enhanced and Web APL Enabled Relational Database for Structural Virology. Nucl. Acids. Res. 2009, 37, D436-D442.

66.Mateo, R; Mateu, M.G. Deterministic, Compensatory Mutational Events in the Capsid of Foot-and-Mouth Disease Virus in Response to the Introduction of Mutations Found in Viruses from Persistent Infections. J. Virol. 2007, 81, 1879-1887.

67.Pérez, R.; Castellanos, M.; Rodríguez-Huete, A; Mateu, M.G. Molecular Determinants of Self-Association and Rearrangement of a Trimeric Intermediate During the Assembly of a Parvovirus Capsid. J. Mol. Biol. 2011, 413, 32-40.

68.Ros, C.; Baltzer, C.; Mani, B.; Kempf, C. Parvovirus Uncoating in Vitro Reveals a Mechanism of DNA Release without Capsid Disassembly and Striking Differences in Encapsidated DNA Stability. J. Virol. 2006, 345, 137-147.

69.Medrano, M.; Fuertes, M.A., Valbuena, A.; Carrillo, P.J.; Rodríguez-Huete, A.; Mateu, M.G. Imaging and Quantitation of a Succession of Transient Intermediates Reveal the Reversible Self-Assembly Pathway of a Simple Icosahedral Virus Capsid. J. Am. Chem. Soc. 2016, 138, 15385-15396.

70.Speir, J.A., Bothner, B., Qu, C., Willits, D.A., Young, M.J., Johnson, J.E. Enhanced Local Symmetry Globally Stabilize a Mutant Virus Capsid That Maintains Infectivity and Capsid Dynamics. J. Virol. 2006, 80, 3582-3591.

71.Carreira, A.; Mateu, M.G. Structural Tolerance Versus Functional Intolerance to Mutation of Hydrophobic Core Residues Surrounding Cavities in a Parvovirus Capsid. J. Mol. Biol. 2006, 360, 1081-1093.

72.Ivanovska, I.L.; de Pablo, P.J.; Ibarra, B.; Sgalari, G.; MacKintosh, F.C.; Carrascosa, J.L.; Schmidt, C.F.; and Wuite, G.J.L. Bacteriophage Capsids: Tough Nanoshells with Complex Elastic Properties. Proc. Natl. Acad. Sci. USA 2004, 101, 7600-7605.

73.Carrasco, C.; Carreira, A.; Schaap, I.A.T.; Serena, P.A.; Gómez-Herrero, J.; Mateu, M.G.; de Pablo, P.J. DNA-Mediated Anisotropic Mechanical Reinforcement of a Virus. Proc. Natl. Acad. Sci. 2006, 103, 13706-13711.

74.Carreira, A.; Menéndez, M.; Reguera, J.; Almendral, J.M.; Mateu, M.G. In Vitro Disassembly of a Parvovirus Capsid and Effect on Capsid Stability of Heterologous Peptide Insertions in Surface Loops. J. Biol. Chem. 2004, 279, 6517-6525.

75.Gardiner, E.M.; Tattersall, P. Mapping of the Fibrotropic and Lymphotropic Host Range Determinants of the Parvovirus Minute Virus of Mice. J. Virol. 1988, 62, 2605-2613. 
76.Moreno-Herrero, F.; Colchero, J.; Gómez-Herrero, J.; Baró, A.M. Atomic Force Microscopy Contact, Tapping, and Jumping Modes for Imaging Biological Samples in Liquids. Phys. Rev. E Stat. Nonlin. Soft Matter Phys. 2004, 69, 031915.

77.Sader, J.E.; Chon, J.W.M.; Mulvaney, P. Calibration of Rectangular Atomic Force Microscope Cantilevers. Review of Scientific Instruments 1999, 70, 3967-3969.

78.Horcas, I.; Fernández, R.; Gómez-Rodríguez, J.M.; Colchero, J.; Gómez-Herrero, J.; Baró, A.M. WSXM: A Software for Scanning Probe Microscopy and a Tool for Nanotechnology. Review of Scientific Instruments 2007, 78, 013705.

79.Sievers, F.; Wilm, A.; Dineen, D.G.; Gibson, T.J.; Karplus, K.; Li, W.; Lopez, R.; McWilliam, H.; Remmert, M.; Söding, J.; Thompson, J.D.; Higgins, D.G. Fast, Scalable Generation of High-Quality Protein Multiple Sequence Alignments Using Clustal Omega. Molecular Systems Biology 2011, 7, 539.

80.Vriend, G. WHAT IF: A Molecular Modeling and Drug Design Program. J. Mol. Graph. $1990,8,52-56$.

81.Humphrey, W.; Dalke, A.; Schulten, K. VMD: Visual Molecular Dynamics. J.Mol.Graph. 1996, 14, 33-38.

82.DeLano, W.L. DeLano Scientific LLC, San Carlos, CA, USA. 2002. Pymol Molecular Graphics System on World Wide Web URL: http://www.pymol.org.

\section{FIGURE LEGENDS}

Figure 1. Structure of MVM (a) Cross-section of the atomic structure of the MVM virion (PDB ID: 1MVM). ${ }^{50}$ ssDNA segments bound to equivalent sites at the capsid inner wall are colored green. (b) Scheme of the icosahedral MVM capsid architecture. S2, S3, S5 labels respectively indicate the position of a 2-fold, 3-fold, 5-fold symmetry axis. Triangular facets and black lines between facets respectively represent idealized trimeric capsid building blocks and intertrimer interfaces. Green ellipses correspond to DNA-binding sites close to 2-fold axes. Small white ellipses correspond to capsid cavities. Small blue circles correspond to capsid pores at the 5-fold axes. (c) Icosahedral model (upper images), crystallographic atomic model (middle images) and AFM images (bottom) of MVM single capsids viewed along a 2fold (S2), 3-fold (S3) or 5-fold (S5) axis. 
Figure 2. Location in the MVM capsid of amino acid residues analyzed in this study. Parts of the atomic structure of the VP2-only MVM capsid (PDB ID: 1Z14) ${ }^{51}$ are represented. (a, b) front (a) and side (b) views of a capsid trimer. (c) front view of a capsid fragment corresponding to five bound trimers surrounding a capsid 5-fold axis. The capsid proteins are represented as backbone models. Residues at the intertrimer interfaces selected for mutational mechanical analysis are depicted as blue spacefilling models, and identified using blue labels. Residues depicted as yellow spacefilling models (yellow labels) form a structurally and functionally discrete cluster the base of the capsid pores at the confluence of five trimers (see text).

Figure 3. Comparison of mechanical stiffness between the wt MVM capsid and engineered capsids in which individual, specific side chains involved in hydrogen bonds between trimeric subunits had been removed by mutation. In each graph, each bar in the histogram represents the number of events (i.e., Fz curves determined) that yielded an elastic constant $k$ within a defined range of values, either for the mutant specified by the label at left (white bars), or for the wt control capsid (black bars). Histograms in the left, middle or right column respectively correspond to $k$ values for $\mathrm{S} 2, \mathrm{~S} 3$ or S5 regions in the capsid.

Figure 4. Comparison of mechanical stiffness between the wt MVM capsid and engineered capsids in which individual, specific side chains involved in non-directional non-covalent interactions between trimeric subunits had been removed by mutation. In each graph, each bar in the histogram represents the number of events (i.e., Fz curves determined) that yielded an elastic constant $k$ within a defined range of values, either for the mutant specified by the label at left (white bars), or for the wt control capsid (black bars). Histograms in the left, middle or right column respectively correspond to $k$ values for S2, S3 or $\mathrm{S} 5$ regions in the capsid.

Figure 5. Variations in mechanical stiffness in different regions in MVM capsids in which specific side chains at intertrimer interfaces were removed. (a) for each mutant capsid, bars indicate the $\%$ increase or decrease in elastic constant $k$ value relative to that of the control wt capsid, at S2 (black bars), S3 (grey bars) or S5 (white bars) regions. Horizontal lines traced at $+16 \%$ increase and $-16 \%$ decrease in $k$ value provide an arbitrary cutoff between smaller and substantial variations in stiffness. Roman number (I-III) indicates the types of interaction removed: I, hydrogen bond; II, ionic interaction; III, van der Waals 
interactions, including C-C contacts leading to a hydrophobic effect. Letter ( $\mathrm{R}$ or E) classifies each capsid mutant according to their mechanical stiffness at S2 regions (the centers of intertrimer interfaces): $\mathrm{R}$ (rigid): strong stiffening ( $\geq+38 \%$ ); E (elastic), moderate or no stiffening $(\leq+25 \%)$. For absolute $k$ values and statistical data see Table 2. (b) for each mutant capsid, bars indicate stiffness anisotropy ratios obtained by dividing $k_{\mathrm{S} 2}$ by either $k_{\mathrm{S} 3}$ (black bars) or $k_{\mathrm{S} 5}$ (white bars). The S2:S3 and S2:S5 mechanical anisotropy ratios of the wt capsid were 1.01 and 1.03, respectively. Original data for mutants I167A and N74A were taken from ref. $^{24}$

Figure 6. Localization of class $R$ and class $E$ residues in a trimer of the MVM capsid. A wall-eyed stereo view of a capsid trimer (backbone model) is shown. Class R or Class E residues are respectively represented as red or cyan spacefilling models. Removal of chemical groups corresponding to the side chains of class $\mathrm{R}$ residues led to large increases in the elastic constant $k$ of S2 regions (the areas closest to the intertrimer interfaces). Removal of chemical groups corresponding to the side chains of class $\mathrm{E}$ residues led to no or moderate increases in the elastic constant $k$ of S2 regions (see text).

Figure 7. Localization in the MVM virion of amino acid residues whose role on mechanical stiffness of the MVM particle has been analyzed. (a, b) front (a) and side (b) views of a capsid trimer. (c) front view of a capsid fragment corresponding to five bound trimers surrounding a capsid 5-fold axis. Spacefilling models are represented. The contribution to the mechanical stiffness of different MVM particle regions of chemical groups corresponding to the side chains of 32 residues per capsid subunit (labeled with different colors) has been determined (this study and refs. ${ }^{23-25}$ ). Mutated residues at the intertrimer interfaces, surrounding the base of the capsid pores, delimiting capsid cavities or at capsidDNA interfaces are respectively represented in cyan, yellow, purple or green spacefilling models. 
Table 1. Capsid mutations chosen for analysis: non-covalent protein-protein interactions removed and effect on virus infectivity

\begin{tabular}{|c|c|c|c|c|c|c|c|}
\hline \multirow{2}{*}{$\begin{array}{l}\text { Mutation } \\
\text { in capsid }\end{array}$} & \multirow{2}{*}{$\begin{array}{l}\text { Contact } \\
\text { class }^{\mathrm{a}}\end{array}$} & \multicolumn{3}{|c|}{$\begin{array}{c}\text { Intertrimer interactions } \\
\text { removed }\end{array}$} & \multicolumn{2}{|c|}{$\begin{array}{l}\text { Intratrimer interactions } \\
\text { removed }\end{array}$} & \multirow{2}{*}{$\begin{array}{l}\text { Infectivity of } \\
\text { virus mutant } \\
\text { relative to } \mathrm{wt}^{\mathrm{d}}\end{array}$} \\
\hline & & H-bond ${ }^{b}$ & Ionic & $\begin{array}{l}\text { van der } \\
\text { Waals }^{\mathrm{c}}\end{array}$ & $\mathrm{H}$ - bond Ionic & $\begin{array}{l}\text { van der } \\
\text { Waals }^{\mathrm{c}}\end{array}$ & \\
\hline $\mathrm{H} 72 \mathrm{~A}$ & I, III & T506,D507 & & $16(7)$ & & & $<8 \times 10^{-7}$ \\
\hline $\mathrm{N} 74 \mathrm{~A}$ & I, III & D507 & & 3 & & & 0.93 \\
\hline R83A & I, III & P566 & & 7 & & & $<5 \times 10^{-6}$ \\
\hline S550A & I, III & Q548(2) & & 8 & & & ND \\
\hline K204A & I, III & N80 & & 3 & & & 1.05 \\
\hline D507A & I,II,III & K166,Y168 & K166 & $8(2)$ & & & $<2 \times 10^{-5}$ \\
\hline $\mathrm{K} 241 \mathrm{~A}$ & II & & D553 & & & & 0.93 \\
\hline F55A & III & & & $6(5)$ & & & $<8 \times 10^{-6}$ \\
\hline $\mathrm{I} 84 \mathrm{~A}$ & III & & & 1 & & $8(7)$ & 0.08 \\
\hline I207A & III & & & $2(2)$ & & & 0.11 \\
\hline N554A & III & & & $5(2)$ & & & ND \\
\hline $\mathrm{I} 167 \mathrm{~A}$ & & & & & & & 0.07 \\
\hline
\end{tabular}

${ }^{a}$ Roman numbers indicates the types of interaction removed in the MVM capsid ${ }^{51}$ (PDB ID: 1Z14): I, hydrogen bond; II, ionic interaction; III, van der Waals interactions, including C-C contacts leading to a hydrophobic effect. The program WHAT $\mathrm{IF}^{80}$ was used with the following cutoff distances: hydrogen bonds: $3.6 \AA$ coulombic interactions: $6 \AA$; van der Waals contacts: $0.8 \AA$ higher than the sum of the van der Waals radii of the atoms considered.

${ }^{\mathrm{b}}$ When more than one hydrogen bond (per subunit) is removed, the number is indicated in parenthesis.

${ }^{\mathrm{c}}$ The number (per subunit) of van der Waals contacts removed and (in parenthesis) the number of C-C contacts removed are indicated.

${ }^{\mathrm{d}}$ Relative infectious titer, calculated by dividing the average absolute titer of each mutant by that of the wt virus in the same experiment, and averaging the results of two experiments. Titers were taken from ref. ${ }^{64} \mathrm{ND}$, not determined, because introduction of these 2 mutations in the MVM infectious clone could not be achieved. 
Table 2. Mechanical stiffness of different regions in MVM capsids in which specific side chains at intertrimer interfaces were removed

\begin{tabular}{|c|c|c|c|c|c|c|c|c|c|c|c|c|}
\hline & \multicolumn{4}{|c|}{ S2 } & \multicolumn{4}{|c|}{$\mathbf{S 3}$} & \multicolumn{4}{|c|}{ S5 } \\
\hline Capsid & $k(\mathrm{~N} / \mathrm{m})^{\mathrm{a}}$ & $F z^{\mathrm{b}}$ & $n^{\mathrm{c}}$ & $p^{\mathrm{d}}$ & $k(\mathrm{~N} / \mathrm{m})^{\mathrm{a}}$ & $F z^{\mathbf{b}}$ & $n^{\mathrm{c}}$ & $p^{\mathrm{d}}$ & $k(\mathrm{~N} / \mathrm{m})^{\mathrm{a}}$ & $F z^{\mathbf{b}}$ & $n^{\mathrm{c}}$ & $p^{\mathrm{d}}$ \\
\hline wt & $0.63 \pm 0.12$ & 41 & 7 & & $0.62 \pm 0.16$ & 52 & 10 & & $0.61 \pm 0.11$ & 24 & 4 & \\
\hline $\mathrm{H} 72 \mathrm{~A}$ & $0.73 \pm 0.12$ & 35 & 5 & $6.0 \times 10^{-4}$ & $0.89 \pm 0.22$ & 18 & 4 & $8.0 \times 10^{-5}$ & $0.63 \pm 0.16$ & 39 & 7 & 0.445 \\
\hline $\mathrm{R} 83 \mathrm{~A}$ & $0.87 \pm 0.19$ & 64 & 7 & $8.8 \times 10^{-13}$ & $0.73 \pm 0.20$ & 47 & 6 & $2.7 \times 10^{-3}$ & $0.81 \pm 0.23$ & 57 & 6 & $6.2 \times 10^{-7}$ \\
\hline S550A & $0.53 \pm 0.12$ & 41 & 7 & $1.8 \times 10^{-4}$ & $0.58 \pm 0.12$ & 34 & 4 & 0.226 & $0.45 \pm 0.07$ & 30 & 3 & $1.6 \times 10^{-6}$ \\
\hline K204A & $0.79 \pm 0.20$ & 47 & 5 & $1.3 \times 10^{-5}$ & $0.64 \pm 0.18$ & 26 & 5 & 0.712 & $0.60 \pm 0.11$ & 58 & 5 & 0.821 \\
\hline D507A & $0.78 \pm 0.20$ & 40 & 6 & $1.5 \times 10^{-4}$ & $0.78 \pm 0.20$ & 46 & 9 & $7.1 \times 10^{-5}$ & $0.70 \pm 0.19$ & 62 & 4 & $4.0 \times 10^{-3}$ \\
\hline K241A & $0.97 \pm 0.27$ & 30 & 4 & $2.2 \times 10^{-7}$ & $0.65 \pm 0.16$ & 36 & 5 & 0.363 & $0.54 \pm 0.13$ & 35 & 4 & 0.046 \\
\hline F55A & $0.95 \pm 0.24$ & 55 & 6 & $1.3 \times 10^{-12}$ & $0.82 \pm 0.21$ & 43 & 6 & $1.5 \times 10^{-6}$ & $0.51 \pm 0.15$ & 61 & 5 & $3.9 \times 10^{-3}$ \\
\hline $\mathrm{I} 84 \mathrm{~A}$ & $0.96 \pm 0.27$ & 43 & 7 & $7.8 \times 10^{-10}$ & $0.84 \pm 0.21$ & 30 & 5 & $8.4 \times 10^{-6}$ & $0.58 \pm 0.15$ & 67 & 5 & 0.498 \\
\hline I207A & $0.96 \pm 0.20$ & 62 & 7 & $2.7 \times 10^{-18}$ & $1.20 \pm 0.22$ & 21 & 5 & $1.3 \times 10^{-11}$ & $0.68 \pm 0.11$ & 26 & 4 & 0.017 \\
\hline $\mathrm{N} 554 \mathrm{~A}$ & $0.77 \pm 0.20$ & 46 & 5 & $1.3 \times 10^{-4}$ & $0.89 \pm 0.19$ & 33 & 3 & $5.3 \times 10^{-9}$ & $0.59 \pm 0.10$ & 71 & 3 & 0.661 \\
\hline $\begin{array}{c}\text { I84A/I207A/ } \\
\text { N554A }\end{array}$ & $1.05 \pm 0.20$ & 38 & 5 & $2.58 \times 10^{-16}$ & $1.19 \pm 0.18$ & 30 & 3 & $5.5 \times 10^{-20}$ & $0.72 \pm 0.22$ & 40 & 3 & $5.6 \times 10^{-3}$ \\
\hline
\end{tabular}

${ }^{\text {a }}$ Elastic constant (average \pm standard deviation)

${ }^{\mathrm{b}}$ Number of indentations used for analysis

${ }^{\mathrm{c}}$ Number of individual particles used for analysis

${ }^{\mathrm{d}} p$-value obtained in a Student t-test with an alpha level $=0.05$ 
Table 3. Mechanical stiffness of S2 and S3 regions in MVM capsids in which specific side chains at interfaces between subunits within a trimer were removed

\begin{tabular}{|c|c|c|c|c|c|c|c|}
\hline \multirow{2}{*}{$\begin{array}{c}\text { Virus } \\
\text { particle }\end{array}$} & \multirow{2}{*}{$\begin{array}{c}\text { Infectivity of } \\
\text { virus mutant } \\
\text { relative to } \\
w^{\mathrm{a}}\end{array}$} & \multicolumn{3}{|c|}{ Capsid stiffness at S3 } & \multicolumn{3}{|c|}{ Capsid stiffness at S2 } \\
\hline & & $k(\mathbf{N} / \mathbf{m})^{\mathbf{b}}$ & $F z^{\mathrm{c}}$ & $p^{\mathrm{d}}$ & $k(\mathbf{N} / \mathbf{m})^{\mathbf{b}}$ & $F z^{\mathrm{c}}$ & $p^{\mathrm{d}}$ \\
\hline wt & 1 & $0.54 \pm 0.14$ & 110 & & $0.55 \pm 0.14$ & 109 & \\
\hline V86A & $3.5 \times 10^{-4}$ & $0.71 \pm 0.43$ & 89 & $4.9 \times 10^{-4}$ & $0.68 \pm 0.30$ & 16 & 0.117 \\
\hline W283A & $<$ treshold & $0.80 \pm 0.38$ & 21 & $1.02 \times 10^{-8}$ & $1.19 \pm 0.21$ & 6 & $2.1 \times 10^{-4}$ \\
\hline Q291A & $5.5 \times 10^{-4}$ & $0.68 \pm 0.17$ & 18 & $4.3 \times 10^{-3}$ & $0.68 \pm 0.18$ & 11 & 0.049 \\
\hline S326A & $<$ treshold & $0.88 \pm 0.32$ & 20 & $1.86 \times 10^{-4}$ & $0.75 \pm 0.14$ & 5 & 0.035 \\
\hline
\end{tabular}

${ }^{a}$ Relative infectious titer, calculated as indicated in Table 1 . Titers were taken from ref. ${ }^{67}<$ treshold means that infectious viruses were below the detection level in plaque assays.

${ }^{\mathrm{b}}$ Elastic constant (average \pm standard deviation)

${ }^{\mathrm{c}}$ Number of indentations used for analysis

${ }^{\mathrm{d}} p$-value obtained in a Student t-test with an alpha level $=0.05$ 